\title{
Uniform convergence of double trigonometric series
}

by

CHANG-PAO CHEN and GWO-BIN CHEN (Hsinchu)

Abstract. It is shown that under certain conditions on $\left\{c_{j k}\right\}$, the rectangular partial sums $s_{m n}(x, y)$ converge uniformly on $T^{2}$. These conditions include conditions of bounded variation of order $(1,0),(0,1)$, and $(1,1)$ with the weights $|j|,|k|,|j k|$, respectively. The convergence rate is also established. Corresponding to the mentioned conditions, an analogous condition for single trigonometric series is

$$
\sum_{|k|=n}^{\infty}\left|\Delta c_{k}\right|=o(1 / n) \quad(\text { as } n \rightarrow \infty) .
$$

For $O$-regularly varying quasimonotone sequences, we prove that it is equivalent to the condition: $n c_{n}=o(1)$ as $n \rightarrow \infty$. As a consequence, our result generalizes those of Chaundy-Jolliffe [CJ], Jolliffe [J], Nurcombe [N], and Xie-Zhou [XZ].

1. Introduction. Let $T^{2} \equiv[-\pi, \pi] \times[-\pi, \pi]$. Consider the double trigonometric series

$$
\sum_{j=-\infty}^{\infty} \sum_{k=-\infty}^{\infty} c_{j k} e^{i(j x+k y)}
$$

where $\left\{c_{j k}:-\infty<j, k<\infty\right\}$ is a double sequence of complex numbers. The rectangular partial sums $s_{m n}(x, y)$ of (1.1) are defined as

$$
s_{m n}(x, y) \equiv \sum_{|j| \leq m|k| \leq n} \sum_{j k} e^{i(j x+k y)} \quad(m, n \geq 0) .
$$

We are interested in finding conditions on $\left\{c_{j k}\right\}$ under which $s_{m n}(x, y)$ converges uniformly on $T^{2}$. This problem for single trigonometric series has been discussed by Chaundy-Jolliffe [CJ], Jolliffe [J], Nurcombe $[\mathrm{N}]$, and

1991 Mathematics Subject Classification: Primary 42A32, 42B05; Secondary 42A20.

Research of the first author is supported by National Science Council, Taipei, R.O.C. under Grant \#NSC 85-2121-M-007-021. 
Xie-Zhou [XZ]. For higher dimensional case, it is still open. Set

$$
\begin{aligned}
& \Delta_{10} c_{j k}=c_{j k}-c_{j+1, k}, \\
& \Delta_{01} c_{j k}=c_{j k}-c_{j, k+1}, \\
& \Delta_{11} c_{j k}=c_{j k}-c_{j+1, k}-c_{j, k+1}+c_{j+1, k+1} .
\end{aligned}
$$

It was proved in [M1] that if both of the conditions

$$
\begin{gathered}
c_{j k} \rightarrow 0 \quad \text { as } \max (|j|,|k|) \rightarrow \infty, \\
\sum_{j=-\infty}^{\infty} \sum_{k=-\infty}^{\infty}\left|\Delta_{11} c_{j k}\right|<\infty,
\end{gathered}
$$

are satisfied, then $s_{m n}(x, y)$ converges pointwise in Pringsheim's sense to some measurable function $f(x, y)$ with $0<|x|,|y| \leq \pi$. Moreover, $f \in$ $L^{p}\left(T^{2}\right)$ for all $0<p<1$, and $s_{m n}(x, y)$ converges in $L^{p}\left(T^{2}\right)$-metric to $f$ as $\min (m, n) \rightarrow \infty$, where

$$
\|f\|_{p} \equiv\left(\frac{1}{4 \pi^{2}} \int_{-\pi}^{\pi} \int_{-\pi}^{\pi}|f(x, y)|^{p} d x d y\right)^{1 / p} \quad(p>0) .
$$

In [CL, M2, M3], it was further proved that $s_{m n}(x, y)$ converges uniformly to $f(x, y)$ on the set $\{\alpha \leq|x| \leq \pi, \beta \leq|y| \leq \pi\}$ for all $0<\alpha, \beta \leq \pi$. As indicated in [C1, C2, M3], $f$ may not be Lebesgue integrable, and hence, condition (1.3) is insufficient for the uniform convergence of $s_{m n}(x, y)$ on $T^{2}$. Instead of (1.3), we assume the following conditions:

$$
\begin{array}{ll}
\text { (1.4) } \sup _{\mu, \nu \geq 0}\left(\sum_{|j| \leq \mu} \sum_{|k| \leq \nu} \chi_{m n}(|j|,|k|) c_{j k}\right) \rightarrow 0 & \text { as } \min (m, n) \rightarrow \infty, \\
\text { (1.5) } \sup _{\mu \geq 1}\left(\mu \sum_{|j|=\mu}^{\infty} \sum_{k=-\infty}^{\infty} \chi_{m n}(|j|,|k|)\left|\Delta_{10} c_{j k}\right|\right) \rightarrow 0 & \text { as } \min (m, n) \rightarrow \infty, \\
\text { (1.6) } \sup _{\nu \geq 1}\left(\nu \sum_{j=-\infty}^{\infty} \sum_{|k|=\nu}^{\infty} \chi_{m n}(|j|,|k|)\left|\Delta_{01} c_{j k}\right|\right) \rightarrow 0 & \text { as } \min (m, n) \rightarrow \infty, \\
\text { (1.7) } \sup _{\mu, \nu \geq 1}\left(\mu \nu \sum_{|j|=\mu}^{\infty} \sum_{|k|=\nu}^{\infty} \chi_{m n}(|j|,|k|)\left|\Delta_{11} c_{j k}\right|\right) \rightarrow 0 & \text { as } \min (m, n) \rightarrow \infty,
\end{array}
$$

where $\chi_{m n}$ denotes the characteristic function:

$$
\chi_{m n}(j, k) \equiv \begin{cases}1 & \text { if }|j|>m \text { or }|k|>n, \\ 0 & \text { otherwise. }\end{cases}
$$

We shall prove in $\S 2$ that these conditions are sufficient for the uniform convergence of $s_{m n}(x, y)$ on $T^{2}$. The convergence rate of $s_{m n}$ is also established there.

Conditions (1.4)-(1.7) are growth conditions on $\left\{c_{j k}\right\}$ with $(j, k)$ lying outside the rectangle $[-m, m] \times[-n, n]$. It is obvious that the $u$-convergence of series (1.1) with $x=y=0$ implies condition (1.4), and (1.4) is stronger than the convergence of the same series in Pringsheim's sense (cf. [D]). Moreover, a double sine series automatically satisfies condition (1.4). For the one-dimensional case, (1.4) reduces to

$$
\sup _{\nu>n}\left(\sum_{n<|k| \leq \nu} c_{k}\right) \rightarrow 0 \quad(\text { as } n \rightarrow \infty) \text {, }
$$

which is equivalent to

$$
\sum_{k=1}^{\infty}\left(c_{k}+c_{-k}\right) \text { converges. }
$$

This is a necessary condition for the uniform convergence. Next, (1.5)-(1.7) are conditions of bounded variation of order $(1,0),(0,1)$, and $(1,1)$ with the weights $j, k, j k$, respectively (cf. Corollary 2.3). Any of them implies (1.3). They are necessary for certain cases, in particular, for $O$-regularly varying quasimonotone sequences (cf. $\S 4$ for details). Corresponding to (1.5)-(1.7), an analogous condition for single trigonometric series is

$$
\sum_{|k|=n}^{\infty}\left|\Delta c_{k}\right|=o(1 / n) \quad(\text { as } n \rightarrow \infty),
$$

where $\Delta c_{k}=c_{k}-c_{k+1}$. It takes the following form for the one-sided condition:

$$
\sum_{k=n}^{\infty}\left|\Delta c_{k}\right|=o(1 / n) \quad(\text { as } n \rightarrow \infty)
$$

For O-regularly varying quasimonotone sequences, we shall prove in Theorem 4.2 that (1.11) is equivalent to.

$$
\lim _{n \rightarrow \infty} n c_{n}=0 \text {. }
$$

This leads us to an alternative approach to the uniform convergence problem. Our result generalizes $[\mathrm{CJ}, \mathrm{J}, \mathrm{N}, \mathrm{XZ}]$

2. Double trigonometric series. As in [C1], define $\Delta_{10}^{*} c_{j k}, \Delta_{01}^{*} c_{j k}$, and $\Delta_{11}^{*} c_{j k}$ by

$$
\begin{gathered}
\Delta_{10}^{*} c_{j k}=c_{j k}-c_{\tau(j), k}, \quad \Delta_{01}^{*} c_{j k}=c_{j k}-c_{j, \tau(k)}, \\
\Delta_{11}^{*} c_{j k}=\Delta_{10}^{*} \Delta_{01}^{*} c_{j k}=\Delta_{01}^{*} \Delta_{10}^{*} c_{j k} .
\end{gathered}
$$


Here $c_{0+, k}=c_{0-, k}=c_{0 k}, c_{j, 0+}=c_{j, 0-}=c_{j 0}$, and the function $\tau(j)$ is defined by $\tau(0+)=1, \tau(0-)=-1, \tau(j)=j+1$ for $j \geq 1$, and $\tau(j)=j-1$ for $j \leq-1$. Obviously, $\Delta_{\alpha \beta}^{*} c_{j k}$ are the same as $\Delta_{\alpha \beta} c_{j k}$ for $j, k \geq 0+$. For other cases, they are related in the following way:

$$
\begin{aligned}
& \Delta_{10}^{*} c_{j k}=-\Delta_{10} c_{j-1, k} \quad(j \leq 0-), \\
& \Delta_{01}^{*} c_{j k}=-\Delta_{01} c_{j, k-1} \quad(k \leq 0-) .
\end{aligned}
$$

Lemma 2.1. Assume that (1.2) holds. Then for $m, n>0$, we have

$\left\|\sum_{|j| \leq m|k| \leq n} \sum_{j k} e^{i(j x+k y)}\right\|_{\infty}$

$$
\begin{aligned}
& \leq \sup _{\mu \leq m ; \nu \leq n}\left|\sum_{|j| \leq \mu} \sum_{|k| \leq \nu} c_{j k}\right|+\sup _{\mu \geq 1}\left(9 \pi \mu \sum_{|j|=\mu}^{\infty} \sum_{k=-\infty}^{\infty}\left|\Delta_{10}^{*} c_{j k}\right|\right) \\
& +\sup _{\nu \geq 1}\left(9 \pi \nu \sum_{j=-\infty}^{\infty} \sum_{|k|=\nu}^{\infty}\left|\Delta_{01}^{*} c_{j k}\right|\right) \\
& \quad+\sup _{\mu, \nu \geq 1}\left(81 \pi^{2} \mu \nu \sum_{|j|=\mu}^{\infty} \sum_{|k|=\nu}^{\infty}\left|\Delta_{11}^{*} c_{j k}\right|\right) .
\end{aligned}
$$

Proof. Let $|x| \leq \pi$ and $|y| \leq \pi$. Define $M=\max ([1 /|x|], 1)$ and $N=$ $\max ([1 /|y|], 1)$. Then we have

$$
\left|\sum_{|j| \leq m} \sum_{|k| \leq n} c_{j k} e^{i(j x+k y)}\right| \leq \Sigma_{11}+\Sigma_{12}+\Sigma_{21}+\Sigma_{22}
$$

where

$$
\Sigma_{\alpha \beta} \equiv\left|\sum_{|j| \leq m} \sum_{|k| \leq n} \chi_{M}^{\alpha}(j) \chi_{N}^{\beta}(k) c_{j k} e^{i(j x+k y)}\right|
$$

and $\chi_{M}^{1}$ and $\chi_{M}^{2}$ denote the characteristic functions $\chi_{[-M, M]}$ and $\chi_{\mathbb{R} \backslash[-M, M]}$, respectively. Set

$$
\Sigma_{11}^{\gamma \delta} \equiv\left|\sum_{|j| \leq m} \sum_{|k| \leq n} \chi_{M}^{1}(j) \chi_{N}^{1}(k) c_{j k}\left(e^{i j x}-1\right)^{\gamma}\left(e^{i k y}-1\right)^{\delta}\right| .
$$

We have $\Sigma_{11} \leq \Sigma_{11}^{00}+\Sigma_{11}^{10}+\Sigma_{11}^{01}+\Sigma_{11}^{11}$. Obviously, $\left|e^{i j x}-1\right| \leq \pi M^{-1}|j|$ and

$$
\left|M^{-1} \sum_{|j| \leq m} \chi_{M}^{1}(j) d_{j}\right| \leq \sup _{1 \leq \mu \leq m} \sum_{|j|=\mu}\left|d_{j}\right|
$$

for any sequence $\left\{d_{j}\right\}$ with $d_{0}=0$. The choice $d_{j}=\sum_{|k| \leq n}\left|j c_{j k}\right|$ gives

$$
\Sigma_{11}^{10} \leq \frac{\pi}{M}\left(\sum_{|j| \leq m} \sum_{|k| \leq n} \chi_{M}^{1}(j)\left|j c_{j k}\right|\right) \leq \sup _{\mu \geq 1}\left(\pi \mu \sum_{|j|=\mu}^{\infty} \sum_{|k| \leq n}\left|\Delta_{10}^{*} c_{j k}\right|\right) .
$$

Similarly, we have

$$
\begin{aligned}
& \Sigma_{11}^{01} \leq \sup _{\nu \geq 1}\left(\pi \nu \sum_{|j| \leq m} \sum_{|k|=\nu}^{\infty}\left|\Delta_{01}^{*} c_{j k}\right|\right), \\
& \Sigma_{11}^{11} \leq \sup _{\mu, \nu \geq 1}\left(\pi^{2} \mu \nu \sum_{|j|=\mu}^{\infty} \sum_{|k|=\nu}^{\infty}\left|\Delta_{11}^{*} c_{j k}\right|\right) .
\end{aligned}
$$

The above discussion shows that

$$
\begin{aligned}
\text { (2.2) } \Sigma_{11} \leq & \sup _{\mu \leq m ; \nu \leq n}\left|\sum_{|j| \leq \mu} \sum_{|k| \leq \nu} c_{j k}\right|+\sup _{\mu \geq 1}\left(\pi \mu \sum_{|j|=\mu}^{\infty} \sum_{|k| \leq n}\left|\Delta_{10}^{*} c_{j k}\right|\right) \\
& +\sup _{\nu \geq 1}\left(\pi \nu \sum_{|j| \leq m|k|=\nu} \sum_{\left.\left|\Delta_{01} c_{j k}\right|\right)}^{\infty} \mid \Delta^{*}\right) \\
& +\sup _{\mu, \nu \geq 1}\left(\pi^{2} \mu \nu \sum_{|j|=\mu|k|=\nu}^{\infty} \sum_{11}^{\infty}\left|\Delta_{11}^{*} c_{j k}\right|\right) .
\end{aligned}
$$

To estimate $\Sigma_{12}$, we employ the functions $\Psi_{k}(t)$, which are defined by $\Psi_{0+}(t)=\Psi_{0-}(t)=1 / 2$ and

$$
\begin{aligned}
\Psi_{k}(t) & =1 / 2+e^{i t}+e^{i 2 t}+\ldots+e^{i k t} \\
\Psi_{-k}(t) & =1 / 2+e^{-i t}+e^{-i 2 t}+\ldots+e^{-i k t}
\end{aligned}
$$

where $k \geq 1$. By [C1, Lemma 2], we get $\Sigma_{12} \leq \Sigma_{12}^{00}+\Sigma_{12}^{10}+\Sigma_{12}^{01}+\Sigma_{12}^{11}$, where

$$
\begin{aligned}
& \Sigma_{12}^{\gamma 0} \equiv\left|\sum_{|j| \leq m|k|=0 \pm} \sum_{|k|=0}^{n} \chi_{M}^{1}(j) \Delta_{01}^{*}\left(\chi_{N}^{2}(k) c_{j k}\right)\left(e^{i j x}-1\right)^{\gamma} \Psi_{k}(y)\right|, \\
& \Sigma_{12}^{\gamma 1} \equiv\left|\sum_{|j| \leq m} \sum_{|k|=n} \chi_{M}^{1}(j) \chi_{N}^{2}(\tau(k)) c_{j, \tau(k)}\left(e^{i j x}-1\right)^{\gamma} \Psi_{k}(y)\right| .
\end{aligned}
$$

As inclicated in [C1], we have $\left|\Psi_{k}(y)\right| \leq \pi /|y| \leq 2 \pi N$. It is clear that $\Delta_{01}^{*}\left(\chi_{N}^{2}(k) c_{j k}\right)=0$ for $|k|<N$, and

$$
\Delta_{01}^{*}\left(\chi_{N}^{2}(k) c_{j k}\right)= \begin{cases}\Delta_{01}^{*} c_{j k} & \text { if }|k|>N \\ -c_{j, \tau(k)} & \text { if }|k|=N\end{cases}
$$

Moreover, $\left|e^{i j x}-1\right| \leq \pi M^{-1}|j|$. Based on these, we get

$$
\Sigma_{12}^{0 \delta} \leq \sup _{\nu \geq 1}\left(4 \pi \nu \sum_{|j| \leq m} \sum_{|k|=\nu}^{\infty}\left|\Delta_{01}^{*} c_{j k}\right|\right) \quad(\delta=0,1)
$$




$$
\begin{aligned}
\Sigma_{12}^{1 \delta} & \leq \sup _{\nu \geq 1}\left(\frac{4 \pi^{2} \nu}{M} \sum_{|j| \leq m|k|=\nu} \sum_{|k|}^{\infty} \chi_{M}^{1}(j)\left|j \Delta_{01}^{*} c_{j k}\right|\right) \\
& \leq \sup _{\mu, \nu \geq 1}\left(4 \pi^{2} \mu \nu \sum_{|j|=\mu}^{\infty} \sum_{|k|=\nu}^{\infty}\left|\Delta_{11}^{*} c_{j k}\right|\right) \quad(\delta=0,1) .
\end{aligned}
$$

$$
\Sigma_{22} \leq \sup _{\mu, \nu \geq 1}\left(64 \pi^{2} \mu \nu \sum_{|j|=\mu}^{\infty} \sum_{|k|=\nu}^{\infty}\left|\Delta_{11}^{*} c_{j k}\right|\right) .
$$

Putting (2.1)-(2.2) and (2.5)-(2.7) together, we obtain the desired result.

THEOREM 2.2. If conditions (1.2) and (1.4)-(1.7) are satisfied, then $s_{m n}$ converges uniformly on $T^{2}$ to some continuous function $f$ as $\min (m, n)$ $\rightarrow \infty$. Moreover,

Putting (2.3)-(2.4) together gives

$$
\begin{aligned}
\Sigma_{12} \leq & \sup _{\nu \geq 1}\left(8 \pi \nu \sum_{j=-\infty}^{\infty} \sum_{|k|=\nu}^{\infty}\left|\Delta_{01}^{*} c_{j k}\right|\right) \\
& +\sup _{\mu, \nu \geq 1}\left(8 \pi^{2} \mu \nu \sum_{|j|=\mu}^{\infty} \sum_{|k|=\nu}^{\infty}\left|\Delta_{11}^{*} c_{j k}\right|\right)
\end{aligned}
$$

Like $\Sigma_{12}$, the term $\Sigma_{21}$ satisfies

$$
\begin{aligned}
\Sigma_{21} \leq & \sup _{\mu \geq 1}\left(8 \pi \mu \sum_{|j|=\mu}^{\infty} \sum_{k=-\infty}^{\infty}\left|\Delta_{10}^{*} c_{j k}\right|\right) \\
& +\sup _{\mu, \nu \geq 1}\left(8 \pi^{2} \mu \nu \sum_{|j|=\mu}^{\infty} \sum_{|k|=\nu}^{\infty}\left|\Delta_{11}^{*} c_{j k}\right|\right) .
\end{aligned}
$$

It remains to estimate $\Sigma_{22}$. With the help of [C1, Lemma 3], we get $\Sigma_{22} \leq \Sigma_{22}^{00}+\Sigma_{22}^{10}+\Sigma_{22}^{01}+\Sigma_{22}^{11}$, where

$$
\begin{aligned}
\Sigma_{22}^{00} & \equiv\left|\sum_{|j|=0 \pm}^{m} \sum_{|k|=0 \pm}^{n} \Delta_{11}^{*}\left(\chi_{M}^{2}(j) \chi_{N}^{2}(k) c_{j k}\right) \Psi_{j}(x) \Psi_{k}(y)\right|, \\
\Sigma_{22}^{01} & \equiv\left|\sum_{|j|=0 \pm}^{m} \sum_{|k|=n} \Delta_{10}^{*}\left(\chi_{M}^{2}(j) \chi_{N}^{2}(\tau(k)) c_{j, \tau(k)}\right) \Psi_{j}(x) \Psi_{k}(y)\right|, \\
\Sigma_{22}^{10} & \equiv\left|\sum_{|j|=m} \sum_{|k|=0 \pm}^{n} \Delta_{01}^{*}\left(\chi_{M}^{2}(\tau(j)) \chi_{N}^{2}(k) c_{\tau(j), k}\right) \Psi_{j}(x) \Psi_{k}(y)\right| \\
\Sigma_{22}^{11} & \equiv\left|\sum_{|j|=m \mid} \sum_{|k|=n} \chi_{M}^{2}(\tau(j)) \chi_{N}^{2}(\tau(k)) c_{\tau(j), \tau(k)} \Psi_{j}(x) \Psi_{k}(y)\right| .
\end{aligned}
$$

A similar argument to (2.3)-(2.4) gives

$$
\Sigma_{22}^{\gamma \delta} \leq \sup _{\mu, \nu \geq 1}\left(16 \pi^{2} \mu \nu \sum_{|j|=\mu}^{\infty} \sum_{|k|=\nu}^{\infty}\left|\Delta_{11}^{*} c_{j k}\right|\right) \quad(\gamma, \delta=0,1)
$$

which implies

$$
\begin{aligned}
\left\|s_{m n}-f\right\|_{\infty} \leq & \sup _{\mu, \nu \geq 0}\left|\sum_{|j| \leq \mu} \sum_{|k| \leq \nu} \chi_{m n}(|j|,|k|) c_{j k}\right| \\
& +\sup _{\mu \geq 1}\left(18 \pi \mu \sum_{|j|=\mu} \sum_{k=-\infty}^{\infty} \chi_{m n}(|j|,|k|)\left|\Delta_{10}^{*} c_{j k}\right|\right) \\
& +\sup _{\nu \geq 1}\left(18 \pi \nu \sum_{j=-\infty}^{\infty} \sum_{|k|=\nu}^{\infty} \chi_{m n}(|j|,|k|)\left|\Delta_{01}^{*} c_{j k}\right|\right) \\
& +\sup _{\mu, \nu \geq 1}\left(324 \pi^{2} \mu \nu \sum_{|j|=\mu}^{\infty} \sum_{|k|=\nu}^{\infty} \chi_{m n}(|j|,|k|)\left|\Delta_{11}^{*} c_{j k}\right|\right),
\end{aligned}
$$

where $\chi_{m n}$ is defined by (1.8).

Proof. Let $\lambda_{m n}$ be on the right of (2.8). Then (1.4)-(1.7) imply that $\lambda_{m n} \rightarrow 0$ as $\min (m, n) \rightarrow \infty$. Set $d_{j k}=\chi_{m n}(|j|,|k|) c_{j k}$. For $M>$ $\max (m, n)$, it follows from Lemma 2.1 that

$$
\begin{aligned}
\left\|s_{m n}-s_{M M}\right\|_{\infty} \leq & \left\|\sum_{|j| \leq M} \sum_{|k| \leq M} d_{j k} e^{i(j x+k y)}\right\|_{\infty} \\
\leq & \sup _{\mu, \nu \leq M}\left|\sum_{|j| \leq \mu} \sum_{|k| \leq \nu} d_{j k}\right|+\sup _{\mu \geq 1}\left(9 \pi \mu \sum_{|j|=\mu}^{\infty} \sum_{k=-\infty}^{\infty}\left|\Delta_{10}^{*} d_{j k}\right|\right) \\
& +\sup _{\nu \geq 1}\left(9 \pi \nu \sum_{j=-\infty}^{\infty} \sum_{|k|=\nu}^{\infty}\left|\Delta_{01}^{*} d_{j k}\right|\right) \\
& +\sup _{\mu, \nu \geq 1}\left(81 \pi^{2} \mu \nu \sum_{|j|=\mu}^{\infty} \sum_{|k|=\nu}^{\infty}\left|\Delta_{11}^{*} d_{j k}\right|\right) \leq \lambda_{m n} .
\end{aligned}
$$

Thus, $\left\{s_{M M}\right\}$ forms a Cauchy sequence in $C\left(T^{2}\right)$. Let $f$ be its limit in $C\left(T^{2}\right)$. Then

$$
\left\|s_{m n}-f\right\|_{\infty}=\lim _{M \rightarrow \infty}\left\|s_{m n}-s_{M M}\right\|_{\infty} \leq \lambda_{m n}
$$

which is (2.8). The desired result follows from this. 
It is clear that

$$
\begin{aligned}
& \sup _{\mu \geq 1}\left(\mu \sum_{|j|=\mu}^{\infty} \sum_{k=-\infty}^{\infty} \ldots\left|\Delta_{10} c_{j k}\right|\right) \leq \sum_{|j|=1}^{\infty} \sum_{k=-\infty}^{\infty} \ldots\left|j \Delta_{10} c_{j k}\right|, \\
& \sup _{\nu \geq 1}\left(\nu \sum_{j=-\infty}^{\infty} \sum_{|k|=\nu}^{\infty} \ldots\left|\Delta_{01} c_{j k}\right|\right) \leq \sum_{j=-\infty}^{\infty} \sum_{|k|=1}^{\infty} \ldots\left|k \Delta_{01} c_{j k}\right| \\
& \sup _{\mu, \nu \geq 1}\left(\mu \nu \sum_{|j|=\mu}^{\infty} \sum_{|k|=\nu}^{\infty} \ldots\left|\Delta_{11} c_{j k}\right|\right) \leq \sum_{|j|=1}^{\infty} \sum_{|k|=1}^{\infty} \ldots\left|j k \Delta_{11} c_{j k}\right| .
\end{aligned}
$$

Hence, Theorem 2.2 has the following consequence.

COROLLARY 2.3. Assume that conditions (1.2), (1.4), and the following conditions are satisfied:

$$
\begin{aligned}
& \sum_{j=-\infty}^{\infty} \sum_{k=-\infty}^{\infty}\left|j \Delta_{10} c_{j k}\right|<\infty \\
& \sum_{j=-\infty}^{\infty} \sum_{k=-\infty}^{\infty}\left|k \Delta_{01} c_{j k}\right|<\infty \\
& \sum_{j=-\infty}^{\infty} \sum_{k=-\infty}^{\infty}\left|j k \Delta_{11} c_{j k}\right|<\infty .
\end{aligned}
$$

Then the conclusions of Theorem 2.2 hold.

3. Single trigonometric series. Denote by $s_{n}(t)$ the $n$th partial sum of the single trigonometric series $\sum_{k=-\infty}^{\infty} c_{k} e^{i k t}$. To modify the proofs of Lemma 2.1 and Theorem 2.2, we get the following one-dimensional analogue of Theorem 2.2, which corresponds to the case where $c_{j k}=0$ for $j \neq 0$. In this case, (1.4) is replaced by (1.9), and (1.10) takes the place of (1.5)-(1.7). We leave the proof to the reader.

THEOREM 3.1. Let $\left\{c_{k}\right\}_{k=-\infty}^{\infty}$ be a null sequence of complex numbers. If conditions (1.9)-(1.10) are satisfied, then $s_{n}$ converges uniformly on $T$ to some continuous function $f$. Moreover,

$$
\left\|s_{n}-f\right\|_{\infty} \leq \sup _{\nu>n}\left|\sum_{n<k \leq \nu}\left(c_{k}+c_{-k}\right)\right|+\sup _{\nu>n}\left(18 \pi \nu \sum_{|k|=\nu}^{\infty}\left|\Delta c_{k}\right|\right) .
$$

It is clear that condition (1.9) is weaker than the following condition:

$$
\sum_{k=1}^{\infty}\left|c_{k}+c_{-k}\right|<\infty
$$

To replace (1.9) by (3.1), we get the following analogue of Theorem 3.1. In this case, condition (1.11) takes the place of (1.10). In $\S 4$, we shall relate this result to $O$-regularly varying quasimonotone sequences.

THEOREM 3.2. Let $\left\{c_{k}\right\}_{k=-\infty}^{\infty}$ be a null sequence of complex numbers. If conditions (1.11) and (3.1) are satisfied, then $s_{n}$ converges uniformly on $T$ to some continuous function $f$. Moreover,

$$
\left\|s_{n}-f\right\|_{\infty} \leq \sum_{k>n}\left|c_{k}+c_{-k}\right|+\sup _{\nu>n}\left(18 \pi \nu \sum_{k=\nu}^{\infty}\left|\Delta c_{k}\right|\right)
$$

Proof. The proof is similar to that of Lemma 2.1 and Theorem 2.2. Let $|t| \leq \pi$. Define $N=\max ([1 /|t|], 1)$. For $m>n$, we have

$$
\begin{aligned}
(3.2)\left|s_{n}(t)-s_{m}(t)\right| & =\left|\sum_{n<|k| \leq m} c_{k} e^{i k t}\right| \\
& =\left|\sum_{n<k \leq m}\left(c_{k}+c_{-k}\right) e^{-i k t}+\sum_{n<k \leq m} c_{k}\left(e^{i k t}-e^{-i k t}\right)\right| \\
& \leq \sum_{n<k \leq m}\left|c_{k}+c_{-k}\right|+\Sigma_{1}+\Sigma_{2},
\end{aligned}
$$

where

$$
\Sigma_{\alpha} \equiv\left|\sum_{n<k \leq m} \chi_{N}^{\alpha}(k) c_{k}\left(e^{i k t}-e^{-i k t}\right)\right|
$$

and $\chi_{N}^{1}$ and $\chi_{N}^{2}$ denote the characteristic functions $\chi_{[-N, N]}$ and $\chi_{\mathbb{R} \backslash[-N, N]}$, respectively. Since $\left|e^{i k t}-e^{-i k t}\right| \leq 2|k t| \leq 2 \pi|k| N^{-1}$, we have

$$
\Sigma_{1} \leq \frac{2 \pi}{N}\left(\sum_{n<k \leq m} \chi_{N}^{1}(k)\left|k c_{k}\right|\right) \leq \sup _{\nu>n}\left(2 \pi \nu \sum_{k=\nu}^{\infty}\left|\Delta c_{k}\right|\right) .
$$

For $\Sigma_{2}$, summation by parts yields

$$
\begin{aligned}
\Sigma_{2} \leq & \sum_{n<k \leq m}\left|\Delta\left(\chi_{N}^{2}(k) c_{k}\right)\right| \cdot\left|\Psi_{k}(t)-\Psi_{-k}(t)\right| \\
& +\left|\left\{\sum_{k=m}-\sum_{k=n^{*}}\right\} \chi_{N}^{2}(\tau(k)) c_{\tau(k)}\left(\Psi_{k}(t)-\Psi_{-k}(t)\right)\right| \\
\leq & \sup _{\nu>n}\left(16 \pi \nu \sum_{k=\nu}^{\infty}\left|\Delta c_{k}\right|\right),
\end{aligned}
$$

where $n^{*}=n$ for $n \geq 1$ and $n^{*}=0 \pm$ for $n=0$. Putting (3.2)-(3.4) together gives

$$
\left\|s_{n}-s_{m}\right\|_{\infty} \leq \sum_{k>n}\left|c_{k}+c_{-k}\right|+\sup _{\nu>n}\left(18 \pi \nu \sum_{k=\nu}^{\infty}\left|\Delta c_{k}\right|\right) .
$$


The desired result follows from this inequality, (1.11), and (3.1).

Write $\sum_{k=-\infty}^{\infty} c_{k} e^{i k t}$ in the form $c_{0}+\sum_{k=1}^{\infty}\left(a_{k} \cos k t+b_{k} \sin k t\right)$. Then $a_{k}=c_{k}+c_{-k}, b_{k}=i\left(c_{k}-c_{-k}\right)$, and

$$
. s_{n}(t)=c_{0}+\sum_{k=1}^{n}\left(a_{k} \cos k t+b_{k} \sin k t\right) \quad(n \geq 1) .
$$

In this notation, condition (3.1) becomes

$$
\sum_{k=1}^{\infty}\left|a_{k}\right|<\infty
$$

If $a_{k}$ and $b_{k}$ are real, then

$$
\sum_{k=n}^{\infty}\left|\Delta c_{k}\right|=\frac{1}{2} \sum_{k=n}^{\infty}\left|\Delta a_{k}-i \Delta b_{k}\right| \geq \frac{1}{2} \max \left(\sum_{k=n}^{\infty}\left|\Delta a_{k}\right|, \sum_{k=n}^{\infty}\left|\Delta b_{k}\right|\right) .
$$

This indicates that condition (1.11) implies both of the following two conditions:

$$
\begin{array}{ll}
\sum_{k=n}^{\infty}\left|\Delta a_{k}\right|=o(1 / n) \quad & (\text { as } n \rightarrow \infty), \\
\sum_{k=n}^{\infty}\left|\Delta b_{k}\right|=o(1 / n) \quad(\text { as } n \rightarrow \infty) .
\end{array}
$$

In the following, we shall extend Theorem 3.2 from (1.11) to (3.7).

THEOREM 3.3. Let $\left\{a_{k}\right\}_{k=1}^{\infty}$ and $\left\{b_{k}\right\}_{k=1}^{\infty}$ be null sequences of complex numbers. If conditions (3.5) and (3.7) are satisfied, then $s_{n}$ converges uniformly on $T$ to some continuous function $f$. Moreover,

$$
\left\|s_{n}-f\right\|_{\infty} \leq \sum_{k>n}\left|a_{k}\right|+\sup _{\nu>n}\left(9 \pi \nu \sum_{k=\nu}^{\infty}\left|\Delta b_{k}\right|\right)
$$

Proof. Let $s_{n}^{1}(t)$ and $s_{n}^{2}(t)$ denote the $n$th partial sums of $c_{0}+$ $\sum_{k=1}^{\infty} a_{k} \cos k t$ and $\sum_{k=1}^{\infty} b_{k} \sin k t$, respectively. Condition (3.5) and the Weierstrass M-test ensure the existence of $f_{1} \in C(T)$ such that $s_{n}^{1} \rightarrow f_{1}$ uniformly on $T$. Moreover, $\left\|s_{n}^{1}-f_{1}\right\|_{\infty} \leq \sum_{k>n}\left|a_{k}\right|$. Applying Theorem 3.2 to the sine series, we find $f_{2} \in C(T)$ such that $s_{n}^{2} \rightarrow f_{2}$ uniformly on $T$, and

$$
\left\|s_{n}^{2}-f_{2}\right\|_{\infty} \leq \sup _{\nu>n}\left(9 \pi \nu \sum_{k=\nu}^{\infty}\left|\Delta b_{k}\right|\right) \text {. }
$$

Therefore, $f \equiv f_{1}+f_{2} \in C(T), s_{n}=s_{n}^{1}+s_{n}^{2}$ converges uniformly on $T$ to $f$, and

$$
\begin{aligned}
\left\|s_{n}-f\right\|_{\infty} & \leq\left\|s_{n}^{1}-f_{1}\right\|_{\infty}+\left\|s_{n}^{2}-f_{2}\right\|_{\infty} \\
& \leq \sum_{k>n}\left|a_{k}\right|+\sup _{\nu>n}\left(9 \pi \nu \sum_{k=\nu}^{\infty}\left|\Delta b_{k}\right|\right) .
\end{aligned}
$$

To end this section, we give an example to distinguish our results from the Weierstrass M-test. Let $c_{0}=c_{1}=c_{-1}=0$, and for $k \geq 2, c_{k}=1 /(k \ln k)$, $c_{-k}=-1 /(k \ln k)$. This example satisfies conditions $(1.9)-(1.11)$ and (3.1). Hence, Theorems 3.1 and 3.2 apply for this case. However, $\sum_{k=-\infty}^{\infty}\left|c_{k}\right|=\infty$, so the Weierstrass M-test fails.

4. Application to $O$-regularly varying quasimonotone sequences. In this section, we shall relate Theorem 3.2 to $O$-regularly varying quasimonotone sequences. Our result generalizes [CJ, J, N, XZ].

A sequence $\{R(n)\}_{n=0}^{\infty}$ of positive numbers is said to be O-regularly varying if it is nondecreasing and for some $\lambda>1$,

$$
\limsup _{n \rightarrow \infty} \frac{R([\lambda n])}{R(n)}<\infty
$$

in other words,

$$
\sup _{n} \frac{R([\lambda n])}{R(n)}<\infty
$$

This generalizes the concept of regularly varying sequences introduced in Karamata [K]. Set $\lambda_{1 n}=[\lambda n]$, and define $\lambda_{\nu n}=\left[\lambda \lambda_{\nu-1, n}\right]$ for $\nu \geq 2$. For $\lambda^{*}>\lambda>1$, choose $\nu$ so large that

$$
\frac{\lambda^{*} n}{\lambda^{\nu}}+\frac{1}{\lambda^{\nu-1}}+\ldots+\frac{1}{\lambda} \leq n \quad\left(\text { for all } n \geq \frac{2}{\lambda-1}\right) .
$$

Then for $n \geq 2 /(\lambda-1)$, we have $\left[\lambda^{*} n\right] \leq \lambda_{\nu n}$, and so

$$
\frac{R([\lambda n])}{R(n)} \leq \frac{R\left(\left[\lambda^{*} n\right]\right)}{R(n)} \leq \frac{R\left(\lambda_{1 n}\right)}{R(n)} \cdot \frac{R\left(\lambda_{2 n}\right)}{R\left(\lambda_{1 n}\right)} \cdots \frac{R\left(\lambda_{\nu n}\right)}{R\left(\lambda_{\nu-1, n}\right)} .
$$

Based on this inequality, we see that if condition (4.1) holds for some $\lambda>1$, then it is satisfied by all $\lambda>1$.

As defined in $[\mathrm{XZ}]$, the sequence $\left\{c_{n}\right\}_{n=0}^{\infty}$ is said to be O-regularly varying quasimonotone if for some $\theta_{0} \in[0, \pi / 2)$ and some $O$-regularly varying sequence $\{R(n)\}_{n=0}^{\infty}$, the following relation holds:

(4.2) $\quad \Delta\left(c_{n} / R(n)\right) \in K\left(\theta_{0}\right) \equiv\left\{z \in \mathbb{C}:|\arg z| \leq \theta_{0}\right\} \quad($ for all $n)$.

We have

$$
\Delta c_{n}+\left(\frac{R(n+1)}{R(n)}-1\right) c_{n}=R(n+1)\left(\Delta \frac{c_{n}}{R(n)}\right)
$$


Hence, condition (4.2) is equivalent to

(4.3) $\quad \Delta c_{n}+\left(\frac{R(n+1)}{R(n)}-1\right) c_{n} \in K\left(\theta_{0}\right) \quad$ (for all $n$ ).

Notice that the quasimonotone sequences defined in Szász [S] correspond to the case of $R(n)=n^{\alpha}$.

LEMMA 4.1. Let $\left\{c_{n}\right\}_{n=0}^{\infty}$ be an O-regularly varying quasimonotone null sequence. Assume that $\theta_{0}$ and $\{R(n)\}_{n=0}^{\infty}$ are the corresponding angle and O-regularly varying sequence. Then the following assertions hold:

(i) $\left|c_{n}\right| \leq\left(\sec \theta_{0}\right) \operatorname{Re} c_{n}$ for all $n$;

(ii) $\left\{\left(\operatorname{Re} c_{n}\right) / R(n)\right\}_{n=0}^{\infty}$ is nonnegative and nonincreasing;

(iii) $\left|\Delta c_{n}+\left(\frac{R(n+1)}{R(n)}-1\right) c_{n}\right| \leq\left(\sec \theta_{0}\right) \operatorname{Re}\left\{\Delta c_{n}+\left(\frac{R(n+1)}{R(n)}-1\right) c_{n}\right\}$

Proof. (i) was proved in [XZ, Lemma 1]. For (ii), we have $\Delta\left(c_{n} / R(n)\right) \in$ $K\left(\theta_{0}\right)$, so

$$
\frac{\operatorname{Re} c_{n}}{R(n)}-\frac{\operatorname{Re} c_{n+1}}{R(n+1)}=\operatorname{Re}\left(\Delta \frac{c_{n}}{R(n)}\right) \geq 0 .
$$

This indicates that $\left\{\left(\operatorname{Re} c_{n}\right) / R(n)\right\}_{n=0}^{\infty}$ is nonincreasing. On the other hand,

$$
\left|\left(\operatorname{Re} c_{n}\right) / R(n)\right| \leq\left|c_{n}\right| / R(1) \rightarrow 0 \quad \text { as } n \rightarrow \infty \text {. }
$$

Hence, $\left(\operatorname{Re} c_{n}\right) / R(n) \geq 0$. This proves (ii). For (iii), we have

and so for all $n$,

$$
\Delta c_{n}+\left(\frac{R(n+1)}{R(n)}-1\right) c_{n} \in K\left(\theta_{0}\right)
$$

$$
\left|\Delta c_{n}+\left(\frac{R(n+1)}{R(n)}-1\right) c_{n}\right| \leq\left(\sec \theta_{0}\right) \operatorname{Re}\left\{\Delta c_{n}+\left(\frac{R(n+1)}{R(n)}-1\right) c_{n}\right\} \text {. }
$$

This completes the proof.

THEOREM 4.2. Let $\left\{c_{n}\right\}_{n=0}^{\infty}$ be an O-regularly varying quasimonotone null sequence. Then condition (1.11) is equivalent to condition (1.12).

Proof. Obviously, (1.11) implies (1.12). This follows from the inequality

$$
\left|n c_{n}\right| \leq n\left(\sum_{k=n}^{\infty}\left|\Delta c_{k}\right|\right)
$$

Conversely, we assume that (1.12) holds. Choose a positive integer $\lambda>1$ such that (4.1) holds. Set

$$
M \equiv \max \left(\sec \theta_{0}, \sup _{n}\left|\frac{R(\lambda n)}{R(n)}-1\right|\right),
$$

where $\theta_{0}$ is the angle appearing in (4.2). By Lemma 4.1(i), (iii), we get

$$
\begin{aligned}
\left|\Delta c_{k}\right| & \leq\left|\Delta c_{k}+\left(\frac{R(k+1)}{R(k)}-1\right) c_{k}\right|+\left|\left(\frac{R(k+1)}{R(k)}-1\right) c_{k}\right| \\
& \leq M \operatorname{Re}\left\{\Delta c_{k}+2\left(\frac{R(k+1)}{R(k)}-1\right) c_{k}\right\} .
\end{aligned}
$$

Summing up both sides with respect to $k$ gives

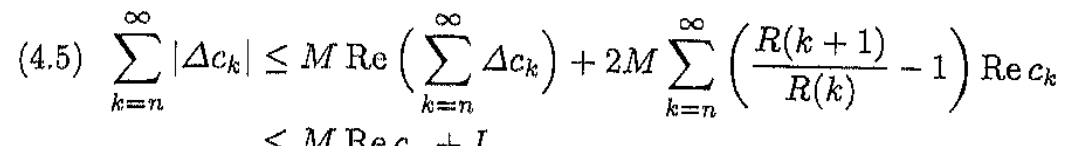

$$
\leq M \operatorname{Re} c_{n}+I
$$

where

$$
I \equiv 2 M\left\{\sum_{k=n}^{\infty}\left(\frac{R(k+1)}{R(k)}-1\right) \operatorname{Re} c_{k}\right\} .
$$

Since $\left(\operatorname{Re} c_{k}\right) / R(k)$ is nonnegative and nonincreasing,

$$
\begin{aligned}
\frac{I}{2 M} & =\sum_{\nu=0}^{\infty}\left\{\sum_{\lambda^{\nu} n \leq k<\lambda^{\nu+1} n}(R(k+1)-R(k)) \frac{\operatorname{Re} c_{k}}{R(k)}\right\} \\
& \leq \sum_{\nu=0}^{\infty} \frac{\operatorname{Re} c_{\lambda^{\nu} n}}{R\left(\lambda^{\nu} n\right)}\left\{\sum_{\lambda^{\nu} n \leq k<\lambda^{\nu+1} n}(R(k+1)-R(k))\right\} \\
& =\sum_{\nu=0}^{\infty} \operatorname{Re} c_{\lambda^{\nu} n}\left(\frac{R\left(\lambda^{\nu+1} n\right)}{R\left(\lambda^{\nu} n\right)}-1\right) \leq M \sum_{\nu=0}^{\infty}\left|c_{\lambda^{\nu} n}\right| \\
& \leq M\left(\sup _{\nu \geq n}\left|\nu c_{\nu}\right|\right)\left(\sum_{\nu=0}^{\infty} \frac{1}{\lambda^{\nu} n}\right) .
\end{aligned}
$$

Putting (1.12), (4.5) and (4.6) together yields

$$
\begin{aligned}
n \sum_{k=2}^{\infty}\left|\Delta c_{k}\right| & \leq M\left|n c_{n}\right|+2 M^{2}\left(\sup _{\nu \geq n}\left|\nu c_{\nu}\right|\right)\left(\sum_{\nu=0}^{\infty} \frac{1}{\lambda^{\nu}}\right) \\
& \rightarrow 0 \quad \text { as } n \rightarrow \infty .
\end{aligned}
$$

This is (1.11) and the proof is complete.

Combining Theorem 4.2 with Theorem 3.2 , we obtain the following result, which generalizes the result [XZ], and hence includes those of ChaundyJolliffe [CJ], Jolliffe $[\mathrm{J}]$, and Nurcombe [N] as special cases. As indicated in $[\mathrm{XZ}]$, condition (1.12) is a necessary condition for $O$-regularly varying quasimonotone sequences. Thus, (1.11) is also needed for such a case.

COROLLARY 4.3. Let $\left\{c_{n}\right\}_{n=-\infty}^{\infty}$ be a null sequence of complex numbers, and suppose that $\left\{c_{n}\right\}_{n=0}^{\infty}$ is an O-regularly varying quasimonotone sequence. 
If conditions (1.12) and (3.1) are satisfied, then $s_{n}$ converges uniformly on $T$ to some continuous function $f$. Moreover,

$$
\left\|s_{n}-f\right\|_{\infty} \leq \sum_{k>n}\left|c_{k}+c_{-k}\right|+18 \pi M^{2} \frac{3 \lambda-1}{\lambda-1} \sup _{\nu>n}\left|\nu c_{\nu}\right|,
$$

where $\lambda>1$ is a positive integer satisfying (4.1) and $M$ is defined by (4.4).

Proof. The first conclusion follows from Theorems 3.2 and 4.2. For the second, Theorem 3.2 gives

$$
\left\|s_{n}-f\right\|_{\infty} \leq \sum_{k>n}\left|c_{k}+c_{-k}\right|+\sup _{m>n}\left(18 \pi m \sum_{k=m}^{\infty}\left|\Delta c_{k}\right|\right) .
$$

For $m>n$, the proof of Theorem 4.2 yields

$$
\begin{aligned}
m \sum_{k=m}^{\infty}\left|\Delta c_{k}\right| & \leq M\left|m c_{m}\right|+2 M^{2}\left(\sup _{\nu \geq m}\left|\nu c_{\nu}\right|\right)\left(\sum_{\nu=0}^{\infty} \frac{1}{\lambda^{\nu}}\right) \\
& \leq M^{2} \frac{3 \lambda-1}{\lambda-1} \sup _{\nu \geq m}\left|\nu c_{\nu}\right| .
\end{aligned}
$$

Combining (4.7) with (4.8), we get the desired estimate for $\left\|s_{n}-f\right\|_{\infty}$.

Acknowledgements. The authors express their gratitude to the referee for his valuable comments on the final version of this paper.

\section{References}

[CJ] T. W. Chaundy and A. E. Jolliffe, The uniform convergence of a certain class of trigonometric series, Proc. London Math. Soc. (2) 15 (1916), 214-216.

[C1] C.-P. Chen, Weighted integrability and $L^{1}$-convergence of multiple trigonometric series, Studia Math. 108 (1994), 177-190.

[C2] - Integrability of multiple trigonometric series and Parseval's formula, J. Math. Anal. Appl. 186 (1994), 182-199.

[CL] C.-P. Chen and C.-C. Lin, Integrability, mean convergence, and Parseval's formula for double trigonometric series, preprint.

[D] M. I. Dyachenko, The rate of u-convergence of multiple Fourier series, Acta Math. Hungar. 68 (1995), 55-70.

[J] A. E. Jolliffe, On certain trigonometric series which have a necessary and sufficient condition for uniform convergence, Math. Proc. Cambridge Philos. Soc. 19 (1921), 191-195.

[K] J. Karamata, Sur un mode de croissance régulière des fonctions, Mathematica (Cluj) 4 (1930), 38-53.

[M1] F. Móricz, Convergence and integrability of double trigonometric series with coefficients of bounded variation, Proc. Amer. Math. Soc. 102 (1988), 633-640.

[M2] -, On the integrability and $L^{1}$-convergence of double trigonometric series, Studia Math. 98 (1991), 203-225.
[M3] F. Móricz, On the integrability of double cosine and sine series $I$, J. Math. Anal. Appl. 154 (1991), 452-465.

[N] J. R. Nurcombe, On the uniform convergence of sine series with quasimonotone coefficients, ibid. 166 (1992), 577-581.

[S] O. Szász, Quasi-monotone series, Amer. J. Math. 70 (1948), 203-206.

[XZ] T. F. Xie and S. P. Zhou, The uniform convergence of certain trigonometric series, J. Math. Anal. Appl. 181 (1994), 171-180.

[Z] A. Zygmund, Trigonometric Series, 2nd ed., Cambridge Univ. Press, Cambridge, 1968.

\section{Department of Mathematics}

National Tsing Hua University

Hsinchu, Taiwan 30043, Republic of China

E-mail: cpchen@math.nthu.edu.tw 